\title{
Talk moves, argumentation, and questioning patterns in LA-supported group problem solving
}

\author{
Alaina Pak, ${ }^{1}$ Marissa Mangini, ${ }^{1}$ Clare Green, ${ }^{1}$ and Tiffany-Rose Sikorski ${ }^{1}$ \\ ${ }^{1}$ The George Washington University, Washington, DC 20052, USA
}

\begin{abstract}
There has been a growing push in universities to increase student participation and discussion as an integral part of the learning process. At least 77 universities have adopted the Colorado-Boulder Learning Assistant Program which trains undergraduate students to facilitate group problem-solving and discussion during class. Prior research on LA-supported clicker question discussions found that when LAs explained answers to students, as they did in $50 \%$ of interactions, group discussion ended. This study further examines LA-student interactions in the context of an introductory physics course. Analysis of LAs' talk moves and questioning patterns revealed that although LAs did not explain answers to students, they favored asking for evidence or reasoning and funneling; these strategies direct students to particular answers as opposed to encourage collaborative sensemaking.
\end{abstract}

\section{INTRODUCTION}

Learning Assistant programs have gained popularity as a way to help faculty increase peer interaction and discussion. Learning Assistants (LAs) are undergraduate students who are hired and trained to support group collaboration and problem-solving in a class that they previously completed successfully [1]. Though multiple studies have confirmed the positive impact of LAs on retention, student satisfaction [2], and conceptual understanding, few studies have actually examined the quality of group work facilitated by LAs.

In one of the first systematic investigations of LA-student interactions, Knight et al. [3] compared LA-supported and unsupported clicker question discussions in an introductory biology course, finding that LA-supported groups were less likely to request information, more likely to request reasoning and feedback, and had longer discussions than groups that did not interact with LAs. However, when LAs tried to explain answers, as they often did, group discussion ended.

Building on the work of Knight et al., this study examines LA-student interactions in an introductory physics course, finding evidence of argumentation, questioning patterns, and Nine Talk Moves. The argumentation codes, developed by Knight $e t a l$. and adapted for the present study, indicate how often LAs and students make claims and ask for or provide reasoning. To elaborate on the Knight et al. "asking questions" code, we looked for three specific questioning patterns: focusing, funneling, and IRE. Finally, we checked for evidence of the Nine Talk Moves, a set of prompts that can be inserted into a conversation to facilitate collaboration. Collectively, the argumentation, questioning patterns, and Nine Talk Moves codes allow for a more detailed account of how LAs and students interact during group problem-solving.

\section{METHODOLOGY}

Data collection occurred for three months of a single semester in an introductory physics with biology applications course required for biomedical engineering majors. The course was supported by four LAs and taught in SCALE-UP format, a studio-style instructional approach that requires am-
TABLE I. Distribution of analyzed recordings.

\begin{tabular}{lccr}
\hline \hline Table & Students (Groups) & Clips & Total Duration \\
\hline Table 1 & $5(2)$ & 20 & $130.05 \mathrm{~min}$ \\
Table 2 & $6(2)$ & 10 & $60.10 \mathrm{~min}$ \\
Table 3 & $6(2)$ & 11 & $56.56 \mathrm{~min}$ \\
\hline Total Coded Set & $17(6)$ & 41 & $255.71 \mathrm{~min}$ \\
\hline \hline
\end{tabular}

ple student talk and collaboration. All four LAs completed or were currently enrolled in a pedagogy course focused on facilitating group discussion and problem-solving, taught by the fourth author. Students $(n=44)$ were assigned by the instructor to small groups of two to four, and then assigned to one of eight tables. Microphones and videocameras were placed at tables to record student conversations. Because a single table sat up to three small groups, recordings captured up to three distinct, simultaneous problem-solving conversations at any given moment, with crosstalk between groups also observed. Data collection occurred at the three tables with all students consenting to participate.

Over 68 hours of audio data were collected; 147 instances of small group work with durations two minutes or longer were extracted for further examination. Five research assistants transcribed all 147 clips using ELAN, toggling between the audio and accompanying video files to ensure that all turns of talk were attributed to the correct speaker. Once audio files were transcribed and checked multiple times, the video was destroyed, consistent with the approved IRB. Of the 147 original discussion clips, we eliminated 27 inaudible clips, 72 clips without LA involvement, and 7 clips collected prior to group re-assignments midway through the semester. The final analyzed data set consisted of 41 clips totaling 255.71 minutes of discussion (see Table I).

Knight et al. suggest that LAs directly influence group discussions. Specifically, during clicker question discussions, LAs tended to explain answers and in doing so, shut down conversation. When LAs used questioning prompts, students responded by explaining reasoning. The aim of the present analysis is to analyze a broader set of discussions that occur in a typical active-pedagogy physics course, with attention 
to LAs' use of strategies introduced in LA training. Thus, three research assistants coded the 41 clips by turn of talk for evidence of argumentation [3], Nine Talk Moves [4], and three basic questioning patterns [5], as explained in the next sections. Here, "turn of talk" or "turn" operationally refers to one speaker's continuous speech (as interpreted by the transcriber) before pausing or being interrupted by the next speaker, e.g., a row in the transcript. Disagreements were resolved through argumentation during the process of coding. Consistency checks included reviewing all turns for a particular code and looking for anomalies (a check for false positives), and random selection of transcripts to recode (a check for false negatives). We coded LA, student, and instructor turns to make comparisons among speaker groups.

\section{RESULTS}

Before delving into results of line-by-line coding, we note a few general features across all 41 audio clips. Typical problem solving activities (e.g., clicker questions, whiteboarding) constituted $78 \%$ of the usable LA-supported clips. Group laboratory work comprised $22 \%$ of analyzed clips. Because of frequent student and LA movement, it is difficult to assess exactly how long each LA spent interacting with groups across the 41 clips. However, clips with LA-student interactions averaged 6 minutes and 17 seconds. No statistically significant difference was observed between LA and total turns of talk per clip, or between LA and LA turns of talk per clip.

As shown in Table II, students spoke most frequently (71\% of turns), followed by LAs (10\% of turns) and the instructor ( $5 \%$ of turns). These percentages serve as a reference point to determine whether students are over- or under-represented across the various codes. Note that a single turn may be tagged with multiple codes; hence, total unique turns is not a sum of the column data. Also note that where percentages do not add to $100 \%$ in Tables II-V, the remaining turns were transcribed and coded but could not be attributed to a speaker.

TABLE II. Frequency of turns by speaker.

\begin{tabular}{rcccc}
\hline \hline Total & LA & Student & Instructor \\
Turns & Turns \\
\cline { 2 - 5 } & Turns & Turns & 21 & 12 \\
Nine Talk Moves & 65 & 22 & 1842 & 182 \\
Argumentation & 2590 & 305 & - & 17 \\
Questioning Patterns & 65 & 46 & 1611 & 88 \\
None of the Above & 2260 & 196 & $3357(71 \%)$ & $251(5 \%)$ \\
\hline Total Unique Turns & 4699 & $478(10 \%)$ & 35 \\
\hline \hline
\end{tabular}

As shown in Table II, several aspects of the discussions that slipped through all three code sets, and may indicate points of interest for future work. These include interruption, trailing off explanations, directives ("Now add 5 and 8"), numerical statements (e.g., "F1 equals...plus 70"), LAs providing students insider information ("I think he's going to have you present"), and nonverbal communication. We did not ask participants for their demographic information, another limitation of the study. Here, we present results on the talk moves, questioning patterns, and argumentation codes, to build upon Knight et al.'s findings, and also demonstrate the need for further study of LA-student interactions during problem-solving discussions.

\section{A. Talk Moves emphasizing collaboration were not observed}

The Nine Talk Moves are prompts that LAs can use to invite, sustain, and deepen students' participation. The Talk Moves are organized under four overarching facilitation goals: (1) Help individual students share, expand and clarify their own thinking (Talk Moves 1-3); (2) Help students listen carefully to one another (Talk Move 2); (3) Help students deepen their reasoning (Talk Moves 4-5); and (4) Help students think with others (Talk Moves 7-9). All LAs and instructors of LA-supported courses receive a one-page handout with all Nine Talk Moves, organized by goal [4]. During training, first-time LAs try different talk moves while working with students, and describe how students respond.

TABLE III. Frequency of Talk Moves.

\begin{tabular}{rcccc}
\hline Talk Move & Total & LA & Student & Instructor \\
\hline 1. Time to think & NA & NA & NA & NA \\
2. Say more... & 1 & 1 & - & - \\
3. So, are you saying... & 2 & 1 & - & - \\
4. Who can rephrase... & 1 & - & - & - \\
5. Asking for evidence... & 60 & 19 & 21 & 12 \\
6. Challenge... & 1 & 1 & - & - \\
7. Agree or disagree... & - & - & - & - \\
8. Add on... & - & - & - & - \\
9. Explain another's idea... & - & - & - & - \\
\hline All Talk Moves & 65 & $22(33 \%)$ & $21(31 \%)$ & $12(18 \%)$ \\
\hline \hline
\end{tabular}

All turns of talk that contained verbatim Talk Moves, or close variants, were coded accordingly. Though the talk moves are designed as tools for instructors, we were curious whether students would, after working with LAs, begin use the talk moves to facilitate their own discussions. Importantly, in order to be coded as a Talk Move, the speaker had to be using the Talk Move in a way that suggested inviting others into the conversation. For example, disagreeing with a peer did not count as an instance of Talk Move 7, but asking peers if they disagree did count. We did not code for Talk Move 1: Time to think because we did not have a reliable way to distinguish different kinds of pauses in the audio clips.

Talk moves were used rarely, appearing in only $1 \%$ (65/4699) of turns (see Table III). Talk Moves were most often used by LAs, slightly more than students, and almost twice as often as the Instructor. By a wide margin, the most 
TABLE IV. Frequency of argumentation codes.

\begin{tabular}{rcccc}
\hline \hline & Total & LA & Student & Instructor \\
Argumentation Code & Turns & Turns & Turns & Turns \\
\hline Acknowledgment & 802 & 59 & 595 & 46 \\
Claim & 226 & 5 & 201 & 1 \\
Background Statement & 491 & 72 & 335 & 40 \\
Asking Questions & 736 & 99 & 492 & 64 \\
Requesting Reasoning & 68 & 21 & 26 & 13 \\
Providing Reasoning & 267 & 49 & 193 & 18 \\
\hline All Argumentation & 2590 & $305(12 \%)$ & $1842(71 \%)$ & $182(7 \%)$ \\
\hline \hline
\end{tabular}

commonly used Talk Move was 5. Asking for Evidence or Reasoning, followed by 3. So, are you saying..., 2. Say more..., and 6. Challenge or Counterexample. These talk moves are part of Goals 1 and 3, which focus on individual students' ideas and reasoning. Interestingly, talk moves associated with collaboration (Talk Moves 4, 7, 8 and 9) were nearly absent from our data set. The Talk Moves developers similarly observed infrequent use of Goal 2 and 4 talk moves, relative to Goals 1 and 3, among elementary teachers [6].

\section{B. LAs make few claims and many background statements}

In contrast to the Talk Moves, the Toulmin-inspired Knight et al. codes for argumentation appeared frequently, applied to $64 \%(305 / 478)$ of LA turns, $55 \%$ of student turns (1842/3357), and 73\% (182/251) of instructor turns. As shown in Table IV, the acknowledgement and asking questions codes were most prevalent. An acknowledgment is a response to an utterance that does not add new meaning other than signaling the utterance was heard (see Table VI, line 60). Asking questions, a combination of Knight et al.'s asking questions and prompting questions, refers to a request for basic information, an answer, confirmation, or reasoning, such as Table VI, lines 53 and 67. Background statements refer to factual claims about the problem (see Table VI, line 50). A claim is a statement expressing the speaker's answer for a problem or task, for example Table VI, line 69. We combined Knight et al.'s providing reasoning and using reasoning into providing/using reasoning, applied when a speaker states logic or evidence, usually for a claim but not exclusively (see Table VI, line 75). Requesting reasoning is pressing (e.g.,"explain your reasoning behind it") or inquiring (e.g.,"okay so why B?") for another speaker's logic or evidence for a previous statement. We omitted the Knight et al. "end of discussion" code because the instructor usually indicated when discussions should end.

As shown in Table IV, LAs most frequently asked questions (99 turns), followed by making background statements (72 turns), acknowledgments (59 turns), providing reasoning (49 turns), and requesting reasoning (21 turns). LAs made a claim, essentially stating the answer, five times. The ratio of
TABLE V. Frequency of questioning patterns.

\begin{tabular}{rcccc}
\hline \hline $\begin{array}{r}\text { Questioning } \\
\text { Pattern }\end{array}$ & $\begin{array}{c}\text { Total } \\
\text { Turns }\end{array}$ & $\begin{array}{c}\text { LA } \\
\text { Turns }\end{array}$ & $\begin{array}{c}\text { Student } \\
\text { Turns }\end{array}$ & $\begin{array}{c}\text { Instructor } \\
\text { Turns }\end{array}$ \\
\hline IRE & 16 & 14 & - & 2 \\
Funneling & 44 & 30 & - & 14 \\
Focusing & 3 & 2 & - & 1 \\
\hline All Three Patterns & 65 & $46(71 \%)$ & - & $17(26 \%)$ \\
\hline \hline
\end{tabular}

claims made to reasoning provided was 5:49, indicating that LAs may provide reasoning in attempt to guide students towards the correct answer, without giving it straightaway. LAs made many background statements, perhaps trying to draw students' attention to salient aspects of the problem.

\section{The focusing pattern was rarely used}

During training, LAs at the data collection site are introduced to three questioning patterns: focusing, funneling, and IRE [5]. IRE stands for initiate-respond-evaluate, when for example an LA asks a question, the student replies, and the LA replies with a judgment of correctness (see Table VI, lines 49-51). The funneling pattern is a series of questions that direct students' attention to the LAs' solution path, such as when LAs are trying to avoid explicitly providing students the answer but still want to "guide" students to the answer (see Table VI, lines 49-53). Finally, the focusing pattern directs students' attention to their own reasoning, independent of whether that reasoning is leading to the "correct answer." For example, to focus on the ideas raised in Table VI, lines 50 and 52, the LA could have asked in line 53, "What are ways we can manipulate that equation?" We had hoped to see ample focusing, but expected that IRE or funneling would predominate given our own experiences as LAs (first three authors) and LA instructor (fourth author).

Indeed, of the three questioning patterns, LAs used funneling most often, and focusing rarely (see Table V). However, a comparison of Table IV and Table V reveals that many LA, student, and instructor questions do not fall under any of the three questioning patterns. For example, of the 99 LA turns coded asking questions, only 20 were also coded as focusing, funneling, or IRE, suggesting that LAs generally did not ask extended sequences of questions that pressed on a particular line of reasoning. Instead, LAs asked questions, for example, to redistribute materials ("Can I take that?"), or to check progress ("Are you guys done with the calibration piece?").

An exception is shown in Table VI, where an LA repeatedly presses students for reasoning about an application of Poiseuille's Law. The question asks, "As cholesterol on the walls of the aorta increases, a partial blockage develops that reduces the inner diameter of the aorta by a factor of 2 . We know that without viscosity, the pressure in the section will decrease. What really happens to the pressure drop across 
TABLE VI. LA asks for reasoning and employs funneling pattern.

\begin{tabular}{|c|c|}
\hline Line $\mathrm{Sp}$ & Transcript [Codes Applied] \\
\hline 49 LA & $\begin{array}{l}\text { so what is something new that we learned today? [ask- } \\
\text { ing questions, funneling, IRE] }\end{array}$ \\
\hline $50 \mathrm{~S} 3$ & $\begin{array}{l}\text { well we learned the (inaudible) like this equation [back- } \\
\text { ground statement, inaudible] }\end{array}$ \\
\hline $51 \mathrm{LA}$ & Right [acknowledgment, IRE] \\
\hline $52 \mathrm{~S} 3$ & and like trying to manipulate it [background statement] \\
\hline 53 LA & so what's changing? [asking questions, IRE, funneling] \\
\hline $54 \mathrm{~S} 3$ & radius [background statement] \\
\hline $55 \mathrm{LA}$ & In that equation. [IRE] \\
\hline 56 LA & Right \\
\hline $57 \mathrm{~S} 3$ & And the volume [background statement] \\
\hline $58 \mathrm{LA}$ & Um [LA turn] \\
\hline 59 LA & $\begin{array}{l}\text { yeah but just look at the one side of the equation. [ac- } \\
\text { knowledgment] }\end{array}$ \\
\hline $60 \mathrm{~S} 3$ & okay that helps [acknowledgment] \\
\hline$\ldots \quad \cdots$ & $\ldots$ \\
\hline 67 S4 & $\begin{array}{l}\text { where's you get } \mathrm{R} \text { equals that? [asking questions, Talk } \\
\text { Move 5] }\end{array}$ \\
\hline $68 \mathrm{~S} 3$ & that's just that equation [background statement] \\
\hline 69 S3 & 16 divided by 8 is 2 so that'd be A [claim] \\
\hline $70 \mathrm{LA}$ & $\begin{array}{l}\text { I don't und- where are you getting all these numbers? } \\
\text { [requesting reasoning, asking questions, Talk Move 5] }\end{array}$ \\
\hline $71 \mathrm{~S} 3$ & $\mathrm{Um}$ \\
\hline 72 LA & you're just making up numbers \\
\hline 73 S3 & oh I am just making them up yeah \\
\hline 74 LA & I don't know why \\
\hline
\end{tabular}

that constricted section? Increases by a factor of... (A) 2, (B) 4, (C) 8, (D) 16, or (E) 32." Prior to the transcript shown in Table VI, the LA approaches and asks students why they picked answer B. S5 responds, but the reasoning is not fully elaborated ("because um the radius squared"). The LA, trying to direct attention to the correct answer, repeatedly asks about answer D: "D?",“okay who said D?","Okay so who said D?", "Anyone? Anyone?" Students seem to ignore the LA's prompts until finally S5 responds, "I said D at first so" but "I don't know, I think B's correct." None of the students provide reasoning in response to the LA's request ("you have no reasons?"). The LA directly states that answer B is wrong (line 46, not shown), and begins to "funnel" students toward answer D (lines 49 and 53), but then realizes students are "just making up numbers" (line 70-74). After line 74, the LA funnels again, but with more conceptual grounding (LA: "The R is decreasing by $1 / 2$ then it's to the 4th power then the pressure is going to have to increase by how much to compensate?"). S4 answers correctly "16" and the LA moves on to another table.

Though an exception, the clip demonstrates the usefulness of analyzing questioning patterns, as opposed to just counting questions, in LA-supported discussions. The clip also highlights the many factors to consider when assessing LA effectiveness and group problem-solving performance. By two typical measures, the interaction is a success. The students reach the correct answer, and at least one of the students, S6, seems satisfied with the LA's support ("it's just we made it more complicated"). However, the students rely on equation manipulation, with no evidence of linking mathematical and physical entities, or considering the everyday implications of the answer. Furthermore, the students do not hold each other accountable for reasoning; that work is left to the LA.

\section{CONCLUSION}

While LA programs positively impact distal measures such as students' conceptual understanding, retention, and grades, we need to better understand how LAs actually carry out their primary responsiblity-to support group problem solving in class. Despite applying three sets of codes, much of talk slipped through our scheme. Yet, we did made a few important observations. First, LAs rarely gave answers directly to students. Second, LA made background statements, asked funneling questions, and used Talk Move 5, all of which are consistent with trying to help students arrive at answers. Finally, LAs rarely asked focusing questions and never used Talk Moves designed to promote collaboration. Future work might attempt to amplify attention to the goals of listening carefully to one another and thinking together.

\section{ACKNOWLEDGMENTS}

We thank Tyler Dickinson, Shunichi Hagiya, Takeshi Mizumoto, Daisuke Nogiwa, and Kathleen Smith for their collaboration. An earlier version of this paper was presented at NARST 2017, San Antonio, TX. This study was funded by Honda Motor Company and Honda Research Institute Japan.
[1] Otero, Valerie, Steven Pollock, and Noah Finkelstein. Am J of Phy 7811 (2010): 1218-1224.

[2] Talbot, Robert M., Laurel M. Hartley, Katrina Marzetta, and Bryan S. Wee. J of Coll Sci Teach 445 (2015): 24-30.

[3] J. K. Knight, S. B. Wise, J. Rentsch, and E. M. Furtak. CBE Life Sci. Educ. 144 (2015).
[4] TERC (2012). Checklist: Goals for Productive Discussions and Nine Talk Moves.

[5] B. A. Herbal-Eisenmann and M. L. Breyfogle. Mathematics Teaching in the Middle School 109 (2005).

[6] Michaels, S. and O'Connor, C. Socializing intelligence through talk and dialogue, (2015): 347-362. 